\title{
A CADASIL Case Presenting with Progressive Bulbar Palsy Caused by Acute Simultaneous Multiple Subcortical Infarcts
}

\author{
(D) Elif GÖKÇAL ${ }^{1}$, iD Mehmet KOLUKISA¹, id Nihat MUSTAFAYEV1, id Talip ASIL1
}

1Bezmialem Vakıf University Medical Faculty Hospital, Clinic of Neurology, İstanbul, Turkey

\begin{abstract}
Cerebral autosomal dominant arteriopathy with subcortical infarcts and leukoencephalopathy (CADASIL) is an adult-onset inherited small vessel disease of the brain caused by NOTCH3 mutations. Clinical characteristics of CADASIL include recurrent infarctions, migraine with aura, mood disturbances and cognitive impairment. We report a 35 year-old migraine-free patient with unusual presentation with acute simultaneous multiple subcortical infarctions causing progressive bulbar palsy as the initial manifestation. Hepresented with slurred speech progressing to anarthria and bilateral palsy of lower motor cranial nerves within 24 hour. Initial acute subcortical infarcts enlarged and new infarcts developed in bilateral hemispheres. There was diffuse leukoencephalopathy involving temporal horns and the patient had positive familiy history of migraine and young stroke. CADASIL was diagnosed genetically when NOTCH3 mutationwas shown. The patient improved after treatment with liquid hydration and antiplatelet agent. We experienced with the patient that CADASIL may present with unusual progressive manifestations and bilateral simultaneous infarctions. Irrespective of clinical presentation, patients with leukoencephalopathy and positive familiy history should be tested for NOTCH3 mutation.
\end{abstract}

Keywords: CADASIL, simultaneous infarcts, progression, bulbar palsy

\section{Introduction}

Cerebral autosomal dominant arteriopathy with subcortical infarcts and leukoencephalopathy (CADASIL), which is caused by the mutation in NOTCH3 gene, is the most common hereditary small vessel disease of the brain in adults. The main clinical features of this autosomal dominant inherited disease are recurrent transient ischemic attacks and stroke, migraine, mood disturbances and cognitive impairment (1). Transient ischemic attacks and ischemic strokes are the most frequent manifestations in CADASIL, occurring in up to $85 \%$ of patients (2). As being a small vessel disease, most of the ischemic events appears in the form of subcortical infarctions causing lacunar syndrome clinically. The uncommon clinical and radiological manifestations of patients with CADASIL have been rarely reported in the literature. Here, we report a patient with a progressive bulber palsy and acute simultanously multiple subcortical infarctions as the first manifestation of CADASIL.

\section{Case Report}

A 35-year-old male was admitted to the Bezmialem University Emergency Department with a complaint of worsening slurred speech for 6 hours. His past history was unremarkable including drug or substance use. There was no history of consanguineous marriage. His father had had ischemic stroke a few times and he had become bedridden 2 years before his death at 56 yearold age. Also, his two brothers and one sister had history of migraine headache. On admission, his blood pressure was 120/70 $\mathrm{mmHg}$ and heart rate was 82 bpm (regular), and body temperature was $36.8^{\circ} \mathrm{C}$. There was no abnormality in his electrocardiography. The neck was supple and Kernig sign was

Address for Correspondence: Elif GÖKÇAL, Bezmialem Vakıf University Medical Faculty Hospital, Clinic of Neurology, istanbul, Turkey

Phone: +90 5054927902 E-mail: elifdr99@gmail.com ORCID ID: orcid.org/0000-0003-3309-4368

Cite this article as: Gökçal E, Kolukısa M, Mustayev N, Asil T. A CADASIL Case Presenting with Progressive Bulbar Palsy Caused by Acute Simultaneous Multiple Subcortical Infarcts. Bezmialem Science 2019;7(2):177-9 
absent. Neurologically, he was alert and oriented. His speech was severely dyshartric. There was no facial paralysis or no tongue deviation. The soft palate elevation was good bilaterally. There was no sensory or motor deficit in his extremities and cerebellar examination was normal. His complete blood count, liver and kidney function tests, blood glucose, electrolytes and thyroid function tests, and vasculitic panel was normal. Brain Computed Tomography scanning disclosed periventricular chronic ischemic changes, predominantly in frontal regions. Diffusion weighted magnetic resonance imaging (DWI- MRI) revealed multiple hyperintensities in bilateral subcortical white matter with low ADC values (Figure $1 \mathrm{~A}, \mathrm{~B}$ ). Fluid-attenuated inversion recovery (FLAIR) images showed extensive ischemic gliotic changes in centrum semiovale, periventricular and subcortical

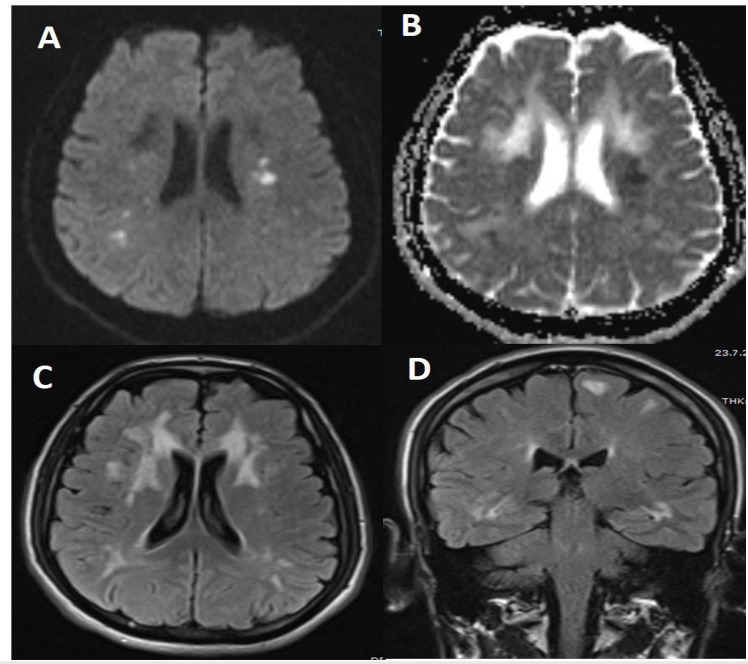

Figure 1. Diffusion Weighted magnetic resonance imaging (DWI- MRI) and FLAIR imagings; multiple hyperintensities in bilateral subcortical white matter with low $A D C$ values $(A, B)$; diffuse leucoencephalopathy involving temporal lobes (C,D)

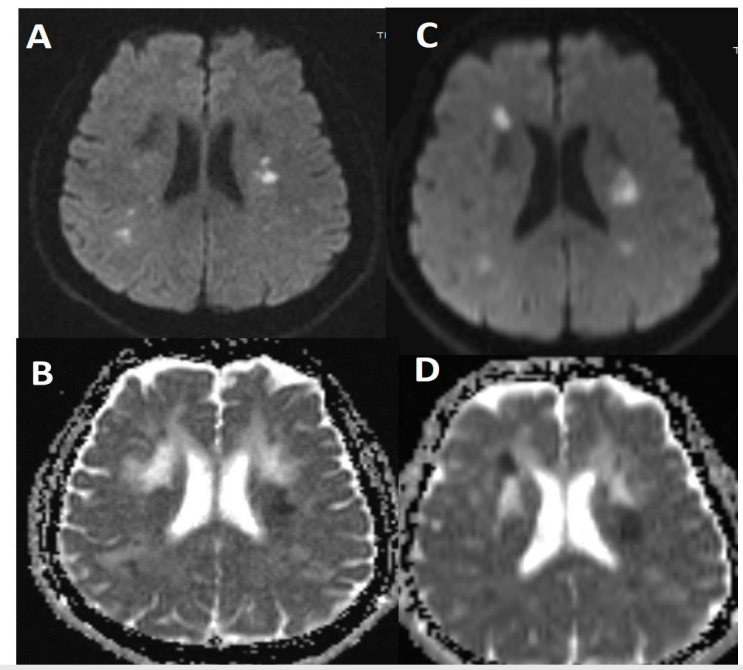

Figure 2. DWI-MRG at onset $(A, B)$ and after clinical progression $(C, D)$ the enlargement of ischemic lesion as well as appearance of new ischemic infarctions in bilateral border-zone areas white matter, basal ganglia, temporal lobes and also in pontin area bilaterally (Figure 1 C, D). He was hospitalized and started dual antiaggregant treatment (asetylsalysililc acid $100 \mathrm{mg}$ and clopidogrel $75 \mathrm{mg}$ daily). 24 hour after his admission, he had a progression with anarthria and bilateral palsy of lower motor cranial nerves. Repeated DWI-MRI demonstrated enlargement of ischemic lesion as well as appearance of new ischemic infarctions in bilateral border-zone areas (Figure 2). There was no hypo-hypertensive episode during follow up period. Extraintracranial CT Angiograhy, transthoracic and transesophageal echocardiography, 24-h Holter ECG analysis were all normal. He improved in 3 weeks with supportive treatment and antiaggregant treatment. Because the patient had diffuse encephalopathy involving temporal lobes and also family history of migraine and stroke at young age, CADASIL was suspected and NOTCH3 gene mutation was found positive.

Informed consent of the patient was taken for publication.

\section{Discussion}

As being a small vessel disease of the brain, ischemic events are almost invariably subcortical and present in $67 \%$ of patients as lacunar syndromes such as pure motor, pure sensory or sensorimotor deficit, ataxic hemiparesis and dysarthria-clumsy hand syndrome in CADASIL (1). Generally, if there is acute simultaneous ischemic infarctions in a patient with stroke, the etiological cause is usually attributed to cardiac or carotid arterial emboli (3). However, etiological work-up did not demonstrate any evidence of embolic etiology in our patient. In the literature, there were 7 cases with CADASIL with multifocal cerebral infarctions and these infarctions were supposed to be related with hemodynamic fluctuations (4-9). One of these reports, global white matter hypoperfusion was demonstrated in MRI perfusion study (6). To the best of our knowledge, there is no case with CADASIL presenting with progressive bulbar palsy probably caused by acute bilateral subcortical lesions in the literature. There was no evidence of hemodynamic failure, as in cases in the published literature, in our patient. The precise pathophysiology of these infarcts remains unclear. In a recent study, small vessel disease of the brain have been proved to be an important cause of acute simultaneous small subcortical infarcts (10).

With this case, we emphasized that CADASIL patients may present with acute simultaneous infarctions and may show a progressive course in the early days. Family history should be taken in detail and brain MRI should be evaluated for diffuse leucoencepholopathy with temporal lobe involvement.

\section{Ethics}

Informed Consent: A consent form was completed by all participants.

Peer-review: Externally peer-reviewed.

\section{Authorship Contributions}

Concept: E.G., N.M., Design: E.G., N.M., Data Collection or Processing: E.G., N.M., Analysis or Interpretation: E.G., N.M., Literature Search: E.G., N.M., Writing: E.G. 
Conflict of Interest: No conflict of interest was declared by the authors.

Financial Disclosure: The authors declared that this study received no financial support.

\section{References}

1. Chabriat H, Joutel A, Dichgans M, Tournier-Lasserve E, Bousser M-G. Cadasil. Lancet Neurol 2009;8:643-53.

2. Bousser M, Tournier-Lasserve E. Cerebral autosomal dominant arteriopathy with subcortical infarcts and leukoencephalopathy: from stroke to vessel wall physiology. J Neurol Neurosurg Psychiatry 2001;70:285-7.

3. Takahashi K, Kobayashi S, Matui R, Yamaguchi S, Yamashita K. The differences of clinical parameters between small multiple ischemic lesions and single lesion detected by diffusion-weighted MRI. Acta Neurol Scand 2002;106:24-9.

4. Gobron C, Viswanathan A, Bousser M-G, Chabriat H. Multiple simultaneous cerebral infarctions in cerebral autosomal dominant arteriopathy with subcortical infarcts and leukoencephalopathy. Cerebrovasc Dis 2006;22:445-6.
5. Saito S, Ozaki A, Takahashi M, Ito H, Matsumoto S, Tomimoto H. Clustering of multifocal cerebral infarctions in CADASIL: a case report. J Neurol 2011;258:325-7.

6. Gordhan A, Hudson BK. Acute watershed infarcts with global cerebral hypoperfusion in symptomatic CADASIL. J Radiol Case Rep 2013;7:8-15.

7. Hsiao C-T, Chen Y-C, Liu Y-T, Soong B-W, Lee Y-C. Acute simultaneous multiple lacunar infarcts as the initial presentation of cerebral autosomal dominant arteriopathy with subcortical infarcts and leukoencephalopathy. J Chin Med Assoc 2015;78:424-6.

8. Taieb G, Renard D, Castelnovo G. Simultaneous subcortical infarcts as initial manifestation of CADASIL. A Acta Neurol Belg 2015;115:385-6.

9. Tojima M, Saito S, Yamamoto Y, Mizuno T, Ihara M, Fukuda H. Cerebral Autosomal Dominant Arteriopathy with Subcortical Infarcts and Leukoencephalopathy with a Novel NOTCH3 Cys323Trp Mutation Presenting Border-Zone Infarcts: A Case Report and Literature Review. J Stroke Cerebrovasc Dis 2016;25:e128-30.

10. Wolf ME, Sauer T, Kern R, Szabo K, Hennerici MG. Multiple subcortical acute ischemic lesions reflect small vessel disease rather than cardiogenic embolism. J Neurol 2012;259:1951-7. 